\title{
Author Correction: Nuclear lamin A/C harnesses the perinuclear apical actin cables to protect nuclear morphology
}

\author{
Jeong-Ki Kim', Arghavan Louhghalam², Geonhui Lee, Benjamin W. Schafer ${ }^{3,4}$, Denis Wirtz ${ }^{3,5,6,7}$ \& \\ Dong-Hwee Kim (D) ${ }^{1}$
}

Correction to: Nature Communications https://doi.org/10.1038/s41467-017-02217-5, published online 14 December 2017

In the original version of this Article, the affiliation details for Arghavan Louhghalam were incorrectly given as 'Institute for NanoBioTechnology, The Johns Hopkins University, Baltimore, MD, 21218, USA', and it should have been given as 'Department of Civil and Environmental Engineering, University of Massachusetts Dartmouth, Dartmouth, MA 02747, USA'. Furthermore, an incorrect grant number, R1610512, was acknowledged. The correct grant number is NRF-2016R1C1B2015018. These errors have now been corrected in both the PDF and HTML versions of the Article.

Published online: 13 March 2018

\begin{abstract}
(c) ()
Open Access This article is licensed under a Creative Commons Attribution 4.0 International License, which permits use, sharing, adaptation, distribution and reproduction in any medium or format, as long as you give appropriate credit to the original author(s) and the source, provide a link to the Creative Commons license, and indicate if changes were made. The images or other third party material in this article are included in the article's Creative Commons license, unless indicated otherwise in a credit line to the material. If material is not included in the article's Creative Commons license and your intended use is not permitted by statutory regulation or exceeds the permitted use, you will need to obtain permission directly from the copyright holder. To view a copy of this license, visit http://creativecommons.org/licenses/by/4.0/.
\end{abstract}

(C) The Author(s) 2018

\footnotetext{
${ }^{1}$ KU-KIST Graduate School of Converging Science and Technology, Korea University, Seoul 02841, South Korea. ${ }^{2}$ Department of Civil and Environmental Engineering, University of Massachusetts Dartmouth, Dartmouth, MA 02747, USA. ${ }^{3}$ Institute for NanoBioTechnology, The John Hopkins University, Baltimore, MD 21218, USA. ${ }^{4}$ Department of Civil Engineering, The John Hopkins University, Baltimore, MD 21218, USA. ${ }^{5}$ Department of Chemical and Biomolecular Engineering, The John Hopkins University, Baltimore, MD 21218, USA. ${ }^{6}$ Johns Hopkins Physical Sciences-Oncology Center, The Johns Hopkins University, Baltimore, MD 21218, USA. ${ }^{7}$ Departments of Pathology and Oncology and Sydney Kimmel Comprehensive Cancer Center, The Johns Hopkins School of Medicine, Baltimore, MD 21205, USA. Correspondence and requests for materials should be addressed to

D.-H.K. (email: donghweekim@korea.ac.kr)
} 